\title{
VARIABLE MAGNITUDE CHARACTERISTICS OF 1-D IIR DISCRETE FILTERS BY A GENERALIZED BILINEAR TRANSFORMATION.
}

\author{
C. S. Gargour \\ Department of Electrical Engineering, \\ École Technologie Supérieure, \\ Montreal, QC, CANADA, H2T 2C8 \\ V. Ramachandran \\ Department of Electrical and Computer \\ Engineering, \\ Concordia University, \\ Montreal, QC, CANADA, H3G 1M8.
}

\begin{abstract}
It is shown that variable magnitude characteristics can be obtained by the application of a general bilinear transformation of the type $s \rightarrow \frac{a_{1} z+a_{0}}{b_{1} z+b_{0}}$ to a known analog
\end{abstract}

filter. Stability considerations dictate the limits of the values of $a_{1}, a_{0}, b_{1}$ and $b_{0}$; and these limits have been determined. Starting from some known filter characteristics in the analog domain, it is shown that different magnitude characteristics can be obtained in the discrete domain.

\section{INTRODUCTION}

The subject of obtaining variable magnitude characteristics in a filter has attracted considerable attention in recent times [see 1-3, for example]. The technique generally used is to start from a known stable filter and then apply a feedback path having a multiplier k. As an alternative, the multiplier can be put in the forward path and the starting filter can be put in the feedback path. In both

\author{
Ravi P. Ramachandran \\ Department of Electrical and Computer \\ Engineering, \\ Rowan University, \\ Glassboro, New Jersey, U.S.A., 08028 \\ and \\ F. Awad \\ Department of Electrical Engineering, \\ École Technologie Supérieure, \\ Montreal, QC, CANADA, H2T 2C8
}

cases, by varying the values of $k$, the magnitude characteristics can be varied. The value of the multiplier $\mathrm{k}$ is determined based on stability considerations. Its limits are governed by the starting filter. In both the cases discussed above, if the starting filter is the same, identical limits of $\mathrm{k}$ are obtained. This treatment can be applied in both one-dimensional (1-D) and two-dimensional (2-D) cases.

A familiar technique of designing a discrete filter is to start from an analog filter and then apply the bilinear transformation

$$
\mathrm{s}=\frac{\mathrm{z}-1}{\mathrm{z}+1}
$$

in order to obtain the discrete transfer function. (This can be applied to 2-D systems also by using bilinear transformations to both the variables).

In this paper, a new approach for obtaining variable magnitude characteristics is given by the generalization of the bilinear transformation given in (1). Specifically, a general bilinear transformation of the type

$$
\mathrm{s}=\frac{\mathrm{a}_{1} \mathrm{z}+\mathrm{a}_{0}}{\mathrm{~b}_{1} \mathrm{z}+\mathrm{b}_{0}}=\mathrm{k} \frac{\mathrm{z}+\mathrm{a}}{\mathrm{z}+\mathrm{b}}
$$

where 


$$
\mathrm{a}=\frac{\mathrm{a}_{0}}{\mathrm{a}_{1}}, \mathrm{~b}=\frac{\mathrm{b}_{0}}{\mathrm{~b}_{1}}, \mathrm{k}=\frac{\mathrm{a}_{1}}{\mathrm{~b}_{1}}
$$

is applied to the starting analog transfer function.

\section{STABILITY CONSIDERATIONS.}

The first problem to be resolved is to obtain the conditions under which stability is maintained. These are given by Theorem 1 .

Theorem 1: The conditions for stability to be maintained when (2) is applied to an analog transfer function are:

$$
\text { (i) }\left|\frac{\mathrm{a}_{0}}{\mathrm{a}_{1}}\right| \leq 1 \text {, (ii) }\left|\frac{\mathrm{b}_{0}}{\mathrm{~b}_{1}}\right| \leq 1 \text {, and (iii) } \mathrm{a}_{0} \mathrm{~b}_{0}<0 \text {. }
$$

Proof: Letting

$$
\begin{array}{ll} 
& s=\sigma+j \omega \\
\text { and } & z=u+j v
\end{array}
$$

in (2), we have

$$
\sigma=\frac{a_{1} b_{1}\left(u^{2}+v^{2}\right)+\left(a_{1} b_{0}+a_{0} b_{1}\right) u+a_{0} b_{0}}{\left(b_{1} u+b_{0}\right)^{2}+\left(b_{1} v\right)^{2}}
$$

and $\quad \omega=\frac{v\left(a_{1} b_{0}-a_{0} b_{1}\right)}{\left(b_{1} u+b_{0}\right)+\left(b_{1} v\right)^{2}}$

For purposes of stability, it is required that for $\sigma=0$,

$$
r^{2}=u^{2}+v^{2} \leq 1
$$

Letting $u=r \cos (\theta)$ in $(4 a)$, the equation to be satisfied will be

$$
a_{1} b_{1} r^{2}+\left(a_{0} b_{1}+a_{1} b_{0}\right) r \cos (\theta)+a_{0} b_{0}=0
$$

The roots of (6) are given by

$$
\mathrm{r}_{1,2}=\frac{1}{2}\left[\begin{array}{l}
-(\mathrm{a}+\mathrm{b}) \cos (\theta) \\
\pm \sqrt{(\mathrm{a}+\mathrm{b})^{2} \cos ^{2}(\theta)-4 a b}
\end{array}\right]
$$

The magnitude of these roots should be less than unity. It can be shown that the only acceptable extrema are located at $\theta$ $= \pm \pi$. The corresponding roots are

$$
\mathrm{r}_{1}= \pm \mathrm{a} \text { and } \mathrm{r}_{2}= \pm \mathrm{b}
$$

This shows clearly $|\mathrm{a}| \leq 1$ and $|\mathrm{b}| \leq 1$.
Also, the requirement $\sigma<0$ for $\mathrm{r}=0$ leads to

$$
\mathrm{a}_{0} \mathrm{~b}_{0}<0 .
$$

Hence the theorem is proved.

Without any loss of generality, one can assume $\mathrm{k}$ in (2) to be positive, which means that $a$ and $b$ should be of opposite signs and

$$
|a| \leq 1 \text { and }|b| \leq 1
$$

Consider the imaginary axis in the analog domain. It can be shown, after some algebraic manipulations, that the condition to be satisfied is

$$
\left(\mathrm{u}+\frac{\mathrm{a}+\mathrm{b}}{2}\right)^{2}+\mathrm{v}^{2}=\frac{(\mathrm{a}-\mathrm{b})^{2}}{4}
$$

This shows that the imaginary axis in the analog domain maps as a circle in the $\mathrm{z}-$ domain with center $\left(-\frac{a+b}{2}+j 0\right)$, which is on the real axis and radius = $\left|\frac{a-b}{2}\right|$. In order that a circle of finite radius is obtained,

$$
a \neq b
$$

Because of the inequalities (10), the radius of this circle is always less than unity. It can be readily verified that the region corresponding to $\sigma<0$ maps into the region inside the circle given by (11). As an illustration, Fig.1 shows a typical region of stability when $\mathrm{a}_{1}=1, \mathrm{a}_{0}=$ $0.5, b_{1}=1$ and $b_{0}=1$. The arcs of circles shown correspond to the circles given by (4b). The stability region is given by a circle having the center at $(-0.25+\mathrm{j} 0)$ and radius $=0.75$. It is clear that only when $a_{1}=1, a_{0}=-1, b_{1}=1, b_{0}=1$, the circle in the $\mathrm{z}$-domain corresponding to the imaginary axis in the analog domain will have unity radius with center at the origin. For all other general bilinear transformations, the circle so obtained in order to ensure stability is contained within this unit circle. 


\section{NUMERICAL EXAMPLE}

As an example, a Butterworth second-order low-pass filter is considered as the starting point. Fig. 2 shows magnitude plots of the transformed transfer functions $\mathrm{k}=2,-1 \leq \mathrm{a} \leq 1$ and $0<\mathrm{b} \leq 1$. The curves marked $\mathrm{xxx}$ correspond to the normalized Butterworth response with $\mathrm{k}=2, \mathrm{a}=-1$ and $b=1$. Four families of curves are given corresponding to four different values of $b$. For each family, the value of ' $a$ ' is varied from -1 to 1 . As can be seen, different magnitude characteristics are obtained.

\section{CONCLUSIONS}

In this paper, it is shown that, by the application of a general bilinear transformation to a starting analog filter transfer function, the magnitude response of 1-D IIR discrete filters can be varied. Though a general bilinear transformation is proposed, stability considerations dictate that certain conditions given by Theorem 1 need be satisfied. Since they are inequalities, infinite number of possibilities exist and therefore, the magnitude characteristics can be varied in a large number of ways. The above treatment can be readily extended to other types of filters like Chebyshev, elliptic, etc., [4]. After the application of the general bilinear transformation to the analog transfer function, the discrete transfer function so obtained can be implemented in varieties of ways.

\section{REFERENCES}

1. C. S. Gargour and V. Ramachandran, "Generation of stable 2-D transfer functions having variable magnitude characteristics", in

MULTIDIMENSIONALSYSTEMS: SIGNAL PROCESSING AND MODELING TECHNIQUES, Academic Press Inc., Vol. 69, 1995, pp.255-297.

2. C. S. Gargour and V. Ramachandran, "Study of the stability and other properties of a class of second order variable magnitude 2-D digital transfer functions with a 2-parameter denominator", IEEE Canadian Conference on Electrical and Computer Engineering, Edmonton, Alberta, Canada, May 1999, pp.683686.

3. C. S. Gargour and V. Ramachandran, "Properties of a class of third-order two-parameter variable magnitude 2D transfer function", $42^{\text {nd }}$ Midwest Symposium on Circuits and Systems, New Mexico State University, August 1999, pp.109-112.

4. M. E. Van Valkenburg, ANALOG FILTER DESIGN, Holt, Rinehart and Winston, 1982. 


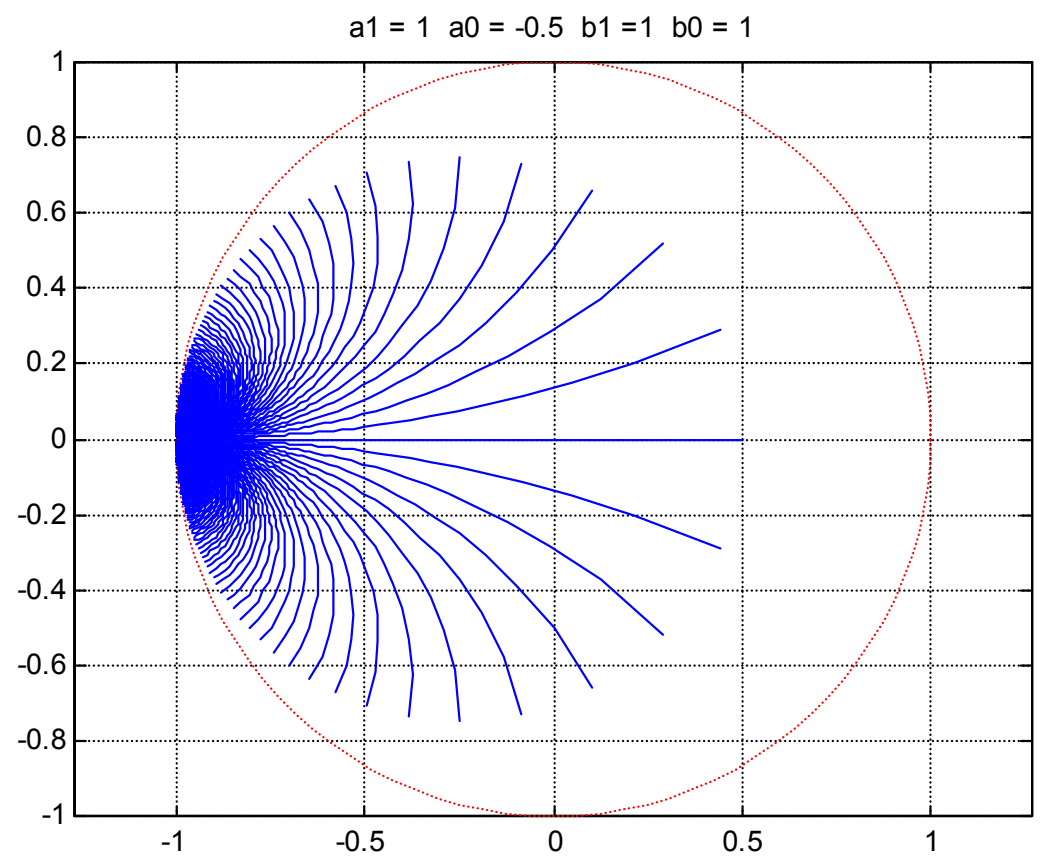

Figure 1: A typical region of stability when $\mathrm{a}_{1}=1, \mathrm{a}_{0}=-0.5, \mathrm{~b}_{1}=1$ and $\mathrm{b}_{0}=1$.

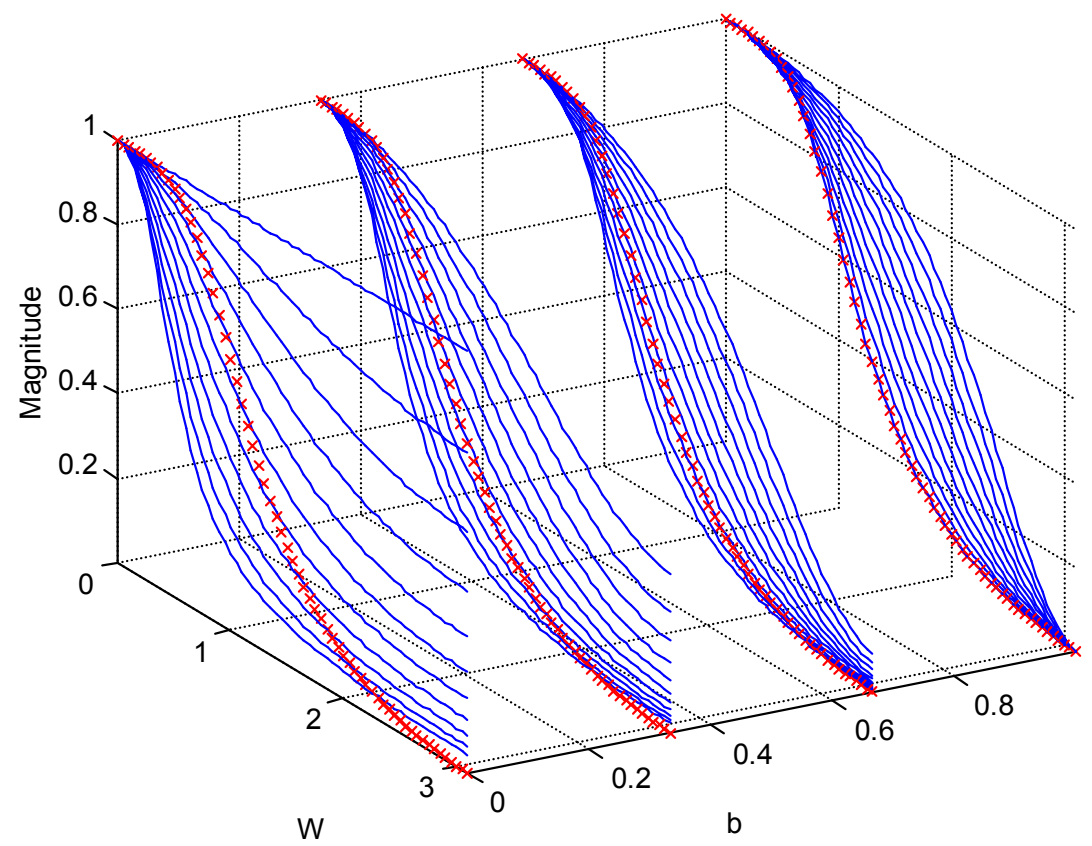

Figure 2: Various Butterworth responses with $\mathrm{k}=2$ and for different values of $\mathrm{a}$ and $\mathrm{b}$. 